
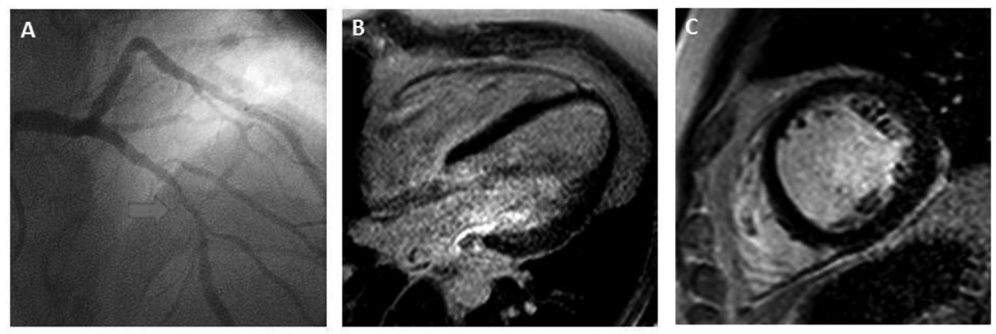

Abstract 15 Figure 1 Case 1- severe disease in mid LAD (arrow) on invasive angiography (A) but no ischaemid scar on LGE imaging (B \& C)

by an independent level 3 CMR reporter. Concordance between ACC and CMR diagnosis of ICM was tested using Cohen's Kappa statistic, with 1 implying complete agreement, and -1 complete disagreement. Net reclassification index was calculated to define how CMR modifies ACC definition of ICM.

Results 147 patients were included in the analysis. Their mean age was 61.3 (SEM 1.1), 79.5\% were male, 19.9\% had NYHA class 3 or 4 symptoms, and LV ejection fraction was $30 \%$ (SEM 1\%). Heart failure therapy included $54 \%$ (SEM 3) of the maximum licenced dose of ACE inhibitor or ARB, 46\% (SEM 3) of the maximum licenced dose of beta-blocker, and $43 \mathrm{mg}$ (SEM 4) furosemide per day. Overall, there was discordance between ACC and CMR definitions in $30(20.4 \%)$ cases. The net reclassification index was $38.3 \%$, with $7 / 62$ cases of ICM diagnosed by CMR being classified by ACC definition as NICM, and 23/85 cases diagnosed as NICM by CMR being defined as per the ACC definition as ICM as demonstrated in case 1. There was significant disagreement between the two methods, with a Cohen's Kappa statistic of 0.596 (SEM 0.064; $\mathrm{p}<0.001)$. Figure (Case1)

Conclusion Classification of ICM is significantly altered by using CMR with patients being reclassified in both directions. Further studies are needed to establish if this improves long term risk stratification in patients with ICM.

Conflict of Interest None

\section{ASSESSING THE ACCURACY OF A NOVEL IN SILICO IMAGING TOOL FOR THE 3D RECONSTRUCTION OF CORONARY VASCULATURE IN THE CONTEXT OF VIRTUAL FRACTIONAL FLOW RESERVE}

Roshni Solanki, Rebecca Gosling, Vignesh Rammohan, Rodney Hose, Patricia Lawford, Julian Gunn, Paul Morris, Yousef Ahmed. University of Sheffield

\subsection{6/heartjil-2019-BCS.15}

Fractional flow reserve (FFR) is the gold standard method for guiding percutaneous coronary intervention. 'Virtual' FFR (vFFR) offers a less-invasive alternative but accuracy is critically dependent on accurate $3 \mathrm{D}$ arterial reconstruction. This is especially challenging with angiography-based solutions due to practical challenges relating to image acquisition, notably table movement between image acquisitions. Some existing methods rely upon restricting table movement, but this poses difficulty in clinical practice. The aim of this study was to validate a novel method for 3D coronary arterial reconstruction under clinically realistic conditions.

Six branched coronary arterial models (3 left and 3 right, 15 vessels) were generated in silico using patient angiograms and 3D printed in PLA (RepRap X400 PRO). All physical models underwent standard coronary angiography imaging. Each model was imaged three times with different restrictions on table movement (18 image datasets, 45 single-vessels). For $3 \mathrm{D}$ reconstruction, vessel centrelines were manually traced on two images $>30^{\circ}$ apart; automatic detection of the borders and diameter optimisation followed (Figure 1). All reconstructions were subjected to vFFR computation. Reconstructions were compared to the reference $3 \mathrm{D}$ files in terms of surface similarity (defined using Hausdorff measurements; averaged distance between a randomised sample of points on both meshes) and physiological analysis (vFFR). The effect of surface reconstruction error on physiological accuracy (vFFR) was described using Pearson's correlation coefficient. To assess accuracy of diameter capture, three aluminium coronary phantoms were fabricated with concentric and eccentric stenoses (diameter range $0.74-1.77 \mathrm{~mm}$, \% narrowing: 44.7-77.2\%). These phantoms also underwent angiography and 3D reconstruction as previously described. Reconstructions were compared with physical micrometer measurements of percentage stenosis and minimum diameter. Accuracy was expressed as mean delta $( \pm S D)$ and absolute error.

Forty-five single-vessel reconstructions were analysed (Figure 2 ). The average distance between reconstructed and reference meshes (reconstruction error) was $0.65 \mathrm{~mm}( \pm 0.30)$ indicating excellent similarity throughout variation of table movement. Mean vFFR was $0.94( \pm 0.049)$ with an average absolute error of $0.008 \pm 0.0098$ and a maximum absolute error of \pm 0.03 . A weak positive relationship between error in reconstruction and physiology was demonstrated $(r=0.370, p=0.013)$. Mean error of stenosis estimation using the metal phantoms was $1.2 \%( \pm 1.2 \%)$. Accuracy of diameter reconstruction at maximum stenosis (minimum diameter) was excellent, with an error of $0.02 \mathrm{~mm}( \pm 0.06 \mathrm{~mm})$.

Coronary anatomy can be reconstructed under realistic conditions with an accuracy that is acceptable for clinical decisionmaking. This novel method has the potential to facilitate interventional decision making as part of a vFFR workflow and may also have value in other areas of anatomical reconstruction.

Conflict of Interest n/a 